\title{
Uma cadeia produtiva mais integrada? A utilização do hedge dinâmico na oscilação dos preços diários da cadeia produtiva da carne suína
}

\author{
A more integrated production chain? The use of dynamic \\ hedge in the daily price oscillation of the pork \\ production chain
}

\author{
Jovani Patias ${ }^{1}$ (D), Sergio Guilherme Schlender ${ }^{1}$ (D), Claudio Edilberto Höfler ${ }^{2}$ (D),
} Marco Antonio da Costa Malheiros ${ }^{2}$ (D) e Leoni Pentiado Godoy ${ }^{1}$

Resumo: Trabalhos recentes têm mostrado uma falta de integração entre o mercado do produtor e o varejo na cadeia produtiva da carne suína. Uma solução para amenizar esse entrave poderia ser feito por meio da estratégia de hedge dinâmico com o modelo Garch-DCC, que permitiria gerenciar as decisões de compra e venda diariamente. Nesse sentido, o objetivo do trabalho foi verificar se o modelo contribui eficientemente com os ajustes de preços diários entre os mercados em comparação ao modelo de regressão linear, fornecendo subsídios aos produtores para se protegerem das oscilações dos preços na cadeia, diminuindo o risco contra grandes variações de preços. Para isso, foi analisado o período de 03/01/2011 a 27/08/2015, em que foram extraídas 1.156 observações para análise. Como resultado, a estratégia de hedge pelo modelo Garch-DCC apresenta melhor desempenho em comparação com a realizada pela Regressão Linear na redução das oscilações do mercado. Com isso, este trabalho encontrou uma abertura para a integração nas percepções e nos processos de negociação desse mercado em análise.

Palavras-chaves: hedge, suinocultura, integração.

Data de submissão: 10 de julho de 2016. Data de aceite: 28 de outubro de 2018.

1. Universidade Federal de Santa Maria (UFSM), Santa Maria (RS), Brasil. E-mails: jovanipatias@gmail.com; sergio.schlender@iffarroupilha.edu.br; leoni_godoy@yahoo.com.br

2. Instituto Federal Farroupilha, Santa Rosa (RS), Brasil. E-mails: claudio.hofler@iffarroupilha.edu.br; marco.malheiros@iffarroupilha.edu.br 
Uma cadeia produtiva mais integrada? A utilização do hedge dinâmico na oscilação dos preços diários da cadeia produtiva da 520 carne suína

\begin{abstract}
Recent work has shown a lack of integration between the producer and retail markets in the pork production chain. A solution to alleviate this obstacle could be performed through the dynamic hedging strategy with the Garch-DCC model, which would allow the management of daily buying and selling decisions. In this sense, the objective of the study was to verify if the model contributes efficiently to the daily price adjustments between the markets in comparison to the linear regression model, providing subsidies to producers to protect themselves from the price oscillations in the chain, reducing the risk against significant changes in prices. In order to do that, the period from 01/03/2011 to 08/27/2015 was analyzed, in which 1,156 observations were extracted for analysis. As a result, the hedge strategy for the Garch-DCC model presents better performance in comparison to the one performed by Linear Regression in reducing market oscillations. With this, this paper found an opening for the integration in the perceptions and the negotiation processes of this market in analysis.
\end{abstract}

Key-words: hedge, pig farming, integration.

Classificação JEL: C30.

\section{Introdução}

Assim como ocorre com todo o setor agropecuário brasileiro, a cadeia da suinocultura passou por importantes avanços na produção de insumos e de animais, bem como nos processos comerciais inerentes à cadeia (Santini \& Souza Filho, 2004). Nessa linha, Rodríguez et al. (2014) afirmam que a gestão da cadeia de abastecimento de carne suína também apresentou transformações no que envolve a coordenação de conjuntos de unidades de explorações agrícolas em diferentes fases de produção.

Assim, no cerne dessa cadeia produtiva, os produtores atuam diretamente no desenvolvimento e na melhoria dos processos, principalmente quando seu grau de interação e conhecimento sobre a cadeia é consistente. Em pesquisa realizada em 2005 pela Embrapa Suínos e Aves (Bassi, 2008), foram analisados os principais canais de acesso a informações agropecuárias de suinocultores e avicultores de base familiar da região Sul do Brasil. Nessa pesquisa, 68\% dos entrevistados tinham baixo nível de escolarização.

Ainda, apenas $10 \%$ possuíam internet em sua propriedade e obtinham informações quanto à produção e à comercialização através de formas tradicionais de repasse de informação (televisão, rádio, entre outros), sendo o contato visual com o gerador ou transmissor da informação um diferencial importante, provado quando a pesquisa aponta que as principais instituições em que o produtor busca informações é a empresa ao qual está integrado $(53,30 \%)$ ou as cooperativas $(40,32 \%)$.
Dessa forma, Santos Filho (2008) afirma que, apesar de a rentabilidade da atividade poder ser explicada por fatores que afetam a oferta e a demanda, o mercado do produtor não se mostrou integrado ao varejo. Esse panorama pode acarretar discrepância entre os entes da cadeia produtiva, como também dos preços trabalhados. Por outro lado, Weydmann \& Seabra (2006) verificam que a cadeia suinícola segue o mesmo padrão de liderança de outras cadeias (com o preço do atacado sendo preço-líder) e que a ineficiência nela fragiliza especialmente o setor produtor.

Assim, Santos (2001) verifica que essa integração por meio de um contrato futuro seria favorável, dado que o mercado da suinocultura apresenta preços muito voláteis e com pouca possibilidade de se utilizar das operações com os preços de outros mercados, como do milho e do boi gordo.

No entanto, a falta de homogeneidade e capacidade de mensurar os preços, o tamanho do mercado físico, o grau de competição do mercado e, principalmente, a utilização de contratos a termo, em que a negociação ocorre em contratos particulares, e não numa bolsa de futuros; bem como os ajustamentos dos seus preços dados no vencimento dos contratos, e não diariamente, são os principais entraves para essa integração (Santos, 2001).

Para corroborar a força dessa integração, Arêdes et al. (2012) verificam que o preço da carne suína em diferentes mercados pode ser ajustado após determinado período, e Martins (2010) verifica que a carne suína do Brasil é fortemente integrada com o preço em termos mundiais, 
sobretudo porque o foco da maior parte da produção é para exportação.

Uma solução para amenizar esses entraves e possibilitar a integração ocorre por meio da estratégia de hedge dinâmico, em que as partes envolvidas nos contratos, em destaque produtores e atacadistas, teriam suas operações de venda e compra ajustadas diariamente, a fim de diminuir o risco contra grandes variações de preços da carne suína. Diferente da estimação por regressão linear, em que o produtor negociaria uma parcela constante da produção física nos preços do atacado, a utilização de uma razão de hedge dinâmico por meio de um modelo DCC-GARCH (Dynamic Conditional Correlation Generalized Autoregressive Conditional Heteroskedasticity) de Engle (2002) permitiria gerenciar as decisões de compra e venda diariamente, através de uma matriz de covariância condicional dos retornos obtidos entre os mercados.

Portanto, o objetivo do presente estudo é analisar se o modelo DCC-GARCH contribui de modo mais eficiente, em comparação ao modelo de regressão linear, com os ajustes de preços diários entre os mercados, fornecendo subsídios aos produtores para se protegerem das oscilações dos preços na cadeia, diminuindo o risco contra grandes variações de preços. Além disso, permitiria um primeiro passo para a integração efetiva do mercado da carne suína, amenizando os principais entraves anteriormente citados.

\section{Fundamentação teórica}

Serão apresentados a seguir os conceitos e as teorias reunidas para a realização deste trabalho de pesquisa, contemplando especificamente a cadeia produtiva da suinocultura.

\subsection{Cadeia produtiva suína}

Ao analisar o desempenho da cadeia produtiva da carne suína, não há como manter uma política de ação eficiente no cenário internacional se os produtos ofertados não forem constantes no nível de qualidade exigidos por esse mercado. Assim, uma administração dos processos produtivos e uma correta distribuição de insumos e produtos devem ser coordenadas ao longo da cadeia para cumprir essas exigências.
No cerne desta contextualização, dada a importância da suinocultura no âmbito nacional e nas particularidades do sistema, a cadeia produtiva divide-se em dois grupos: integrado e independente. No primeiro grupo há coordenação de uma agroindústria ou cooperativa que fornece aos produtores (integrados) insumos, animais, medicamentos e assistência, cabendo ao suinocultor investimentos em manutenção, mão de obra e despesas de custeio, havendo, portanto, uma relação direta entre o suinocultor e as agroindústrias integradas (Miele, 2006). Já o segundo grupo se dá através do chamado mercado spot, no qual as agroindústrias adquirem suínos para abate em suinícolas independentes, que têm liberdade de decisão e de processamento dos animais (Rocha, 2006).

Villwock (2001) aponta que um dos elos mais impactantes na cadeia produtiva está na exigência de garantia alimentar, que está elevando consideravelmente os custos atuais, tanto de produtores como de atacadistas. Para o autor, torna-se necessário, para atender essas exigências legais, a agregação dos processos como a implantação de sistemas de certificação e rastreabilidade dos produtos, desde a utilização dos insumos até o produto final, afetando o nível de competitividade da cadeia.

Além disso, a competitividade da cadeia produtiva está diretamente relacionada com o nível de satisfação do consumidor. Barcellos $(2011,2012)$ apresentam que a produção industrial de alimentos parece ser um sistema aceito, mas que os consumidores estão mostrando que a sustentabilidade ambiental não deve ser esquecida. Ao tratar dos desafios e oportunidades da cadeia produtiva e do consumo de porco no Brasil, Barcellos $(2011,2012)$ citam que, apesar de muitas melhorias tecnológicas da cadeia produtiva suína nos últimos anos, produtores e indústria ainda não estão focados na inovação sobre a informação nem na satisfação do consumidor. Os resultados indicam que os consumidores preferem produtos frescos (não congelados) e de supermercados. No geral, os consumidores de carne suína entrevistados no Brasil sinalizam oportunidades para a cadeia suína em termos de inovação e desenvolvimento de novos produtos. Assim, o melhor atendimento a esses consumidores representa oportunidades para a cadeia produtiva de carne suína.

Por outro lado, a respeito do consumo e da renda, Raimundo \& Batalha (2015) apontam que a carne suína é consumida duas ou três vezes ao mês, tendo sido observado que os homens são consumidores mais frequentes do produto. Os autores ainda ressaltam a 
importância de estudar o comportamento do consumidor final de carne suína, de forma a gerar informações que segmentem esse mercado e possam subsidiar estratégias mercadológicas para as empresas do setor.

Entre alguns estudos relacionados ao mercado suíno brasileiro encontra-se o de Rodrigues et al. (2015), que buscou analisar as participações dos principais estados produtores de carne suína (Minas Gerais, São Paulo, Paraná, Santa Catarina, Rio Grande do Sul, Mato Grosso do Sul, Mato Grosso e Goiás) na produção nacional, em que os resultados obtidos permitiram concluir que a produção suinícola brasileira passou por importantes transformações ao longo da última década, apresentando crescimento.

Já os estudos de Guimarães et al. (2017), por exemplo, cientes da importância do mercado de carne suína e do seu alto consumo, buscaram caracterizar a cadeia produtiva de suínos e seus mercados no Brasil e no mundo, o apoio do BNDES e as principais tendências do setor, em que apontam que as novas descobertas no campo da genética e exigências sanitárias têm alterado significativamente a suinocultura na maior parte do mundo, buscando ampliar seu dinamismo e eficiência, bem como competividade nos preços.

Para tanto, a análise a respeito do impacto dos preços da cadeia da carne suína tem origem no trabalho de Griffith \& Piggott (1994), que verificaram a presença de assimetria no comportamento dos preços no mercado australiano, sobretudo no relacionamento entre o produtor e o preço vendido comercialmente. Além disso, identificou-se que a carne suína é substituta de outras carnes, em destaque a bovina.

Em vista disso, Hayenga et al. (1996) analisam o impacto dos preços de ambos os produtos e verificaram que modelos ligados à volatilidade ajustam melhor a previsão dos preços. No Brasil, Arêdes et al. (2012) também verificaram que a carne suína é produto substituto da bovina. Quando o preço da carne de boi eleva-se, a quantidade consumida tende a diminuir, parte do consumo, então, se desloca para a carne suína.

Baseados no impacto econômico da carne suína, alguns trabalhos estão focados na transmissão de preços dentro da cadeia produtiva da carne suína. Weydmann \& Seabra (2006) identificam a liderança de preços na cadeia da carne suína e os efeitos da instabilidade destes sobre o produtor, o atacado e o varejo da cadeia suinícola de São Paulo no período de 1995 a 2005 . Eles utilizam um modelo de Vetor Autorregressivo (VAR), de um processo de heteroscedasticidade condicional dos autorregressivos (Arch) para a instabilidade dos preços e aplicam o teste de causalidade de Granger para cointegração. Verificam que os preços do atacado são transmitidos ao setor produtor e que a sua variância condicional do atacado é transmitida aos preços do produtor e do varejo e concluem que a cadeia suinícola segue o mesmo padrão de liderança de outras cadeias, com forte influência dos preços pertencentes ao atacado, e que a ineficiência nela fragiliza especialmente o setor produtor.

Martins (2010) aprofunda essa análise de transmissão da cadeia da carne suína e analisa as relações de transmissão dos preços entre os produtores de milho e soja e do preço internacional da carne suína sobre preços recebidos pelos produtores de suínos no Brasil, bem como analisa a integração do mercado internacional de carne suína no período de outubro de 2003 a abril de 2010. Para tanto, usam um modelo autorregressivo com vetor de correção de erro (VEC) e observam que preços recebidos pelos produtores brasileiros de soja, de milho e da carne suína no mercado internacional são relevantes para explicar o comportamento do preço recebido pelos produtores de suínos no Brasil. Além disso, o mercado de carne suína do Brasil apresenta forte integração de preços no mercado mundial dessa carne.

\section{Metodologia}

Considerando-se diferentes particularidades da cadeia produtiva da carne suína, realiza-se pesquisa exploratória de natureza quantitativa (Gil, 2010). Assim, visando estimar a estratégia dinâmica de hedge para a proteção de produtores de carne suína das oscilações do atacado, foram coletados no banco de dados do Instituto de Economia Agrícola (IEA) e no Centro de Pesquisas Avançadas (Cepea) cotações diárias dos preços recebidos pelo produtor e dos preços disponíveis do atacado no estado de São Paulo, correspondentes ao período de 03/01/ 2011 a 27 / 08/2015. O período escolhido, por sua vez, deve-se à disponibilidade conjunta dos dados até o momento de coleta, num total de 1.156 observações diárias.

Para que ocorra execução eficiente da estratégia de hedge, faz-se necessário estimar a cointegração entre os 
preços recebidos e definidos no atacado, a fim de verificar se os preços apresentam movimentos concomitantes no tempo e a diferença que possa existir entre eles seja estável. Nesse sentido, utiliza-se a estimação proposta por Engle \& Granger (1987) para proporcionar equilíbrio de longo prazo. Na hipótese de duas séries não estacionárias formarem um vetor de coeficientes que gerem resíduos estacionários, diz-se que estas séries são cointegradas. Para testar a presença de cointegração, foi efetuado o teste Dickey-Fuller Aumentado (ADF) para as variáveis em nível e nos resíduos. Constatada a presença de cointegração, torna válida a formação de estratégias de hedge entre as séries.

Além disso, a cointegração pressupõe que o ajustamento das diferenças das séries de preços no curto prazo é feito a cada período, e assim segue um padrão linear. Porém, Stigler (2010) verifica que os comportamentos diferenciados de diversas séries econômicas invalidam a hipótese de linearidade. Em vista disso, Hansen \& Seo (2002) propõem um teste para a existência de cointegração linear do modelo, a fim de que se ajuste melhor aos dados. Caso não exista a presença de linearidade, a cointegração linear se dará em diferentes regimes (thresholds).

A cointegração por meio de threshold proposto por Balke \& Fomby (1997) tem como objetivo solucionar as principais críticas existentes contra a cointegração linear, em que o ajustamento não precisa ocorrer instantaneamente, e sim quando os desvios excederem um limiar crítico, cuja origem reside no modelo self-exciting threshold auto-regressive (Setar), de Tong (1990).

Para o presente trabalho, realiza-se a estimação de um modelo vetorial de correção de erro (VECM), que inclui na relação entre o preço recebido pelo produtor e disponível no atacado uma variável que representa os desvios do equilíbrio de longo prazo desses dois preços. Esse modelo visa efetuar ajustes em curto prazo para corrigir os desvios ocorridos no equilíbrio de longo prazo. Após rejeitar a hipótese de cointegração linear através do teste de Hansen \& Seo (2002), foi estimado um modelo TVEC, seguindo a descrição de Stigler (2010), e representado pelas equações 1,2 e 3 .

$$
\left[\begin{array}{c}
\Delta P_{t} \\
\Delta A_{t}
\end{array}\right]=\left\{\begin{array}{l}
{\left[\begin{array}{c}
c_{P}^{L} \\
c_{A}^{L}
\end{array}\right]+\left[\begin{array}{c}
\alpha_{P}^{L} \\
\alpha_{A}^{L}
\end{array}\right] E C T_{t-1}+\beta_{p}^{L}\left[\begin{array}{c}
\Delta P_{t-p} \\
\Delta A_{t-p}
\end{array}\right]+\left[\begin{array}{c}
\varepsilon_{P, t}^{L} \\
\varepsilon_{A, t}^{L}
\end{array}\right](a)} \\
{\left[\begin{array}{c}
c_{P}^{H} \\
c_{A}^{H}
\end{array}\right]+\left[\begin{array}{c}
\alpha_{P}^{H} \\
\alpha_{A}^{H}
\end{array}\right] E C T_{t-1}+\beta_{p}^{H}\left[\begin{array}{l}
\Delta P_{t-p} \\
\Delta A_{t-p}
\end{array}\right]+\left[\begin{array}{c}
\varepsilon_{P, t}^{H} \\
\varepsilon_{A, t}^{H}
\end{array}\right](b)}
\end{array}\right.
$$

$$
\begin{gathered}
E C T_{t-1}=(1,-\beta)\left[\begin{array}{l}
s_{t-1} \\
f_{t-1}
\end{array}\right] \\
{\left[\begin{array}{l}
\Delta P_{t} \\
\Delta A_{t}
\end{array}\right]=\left\{\begin{array}{l}
(a) s e E C T_{t-1} \leq \theta_{L} \\
(b) s e E C T_{t-1} \leq \theta_{L}
\end{array}\right.}
\end{gathered}
$$

Nessas equações, o termo de correção de erro é separado em dois regimes, uma de baixa (L) e a outra de alta $(\mathrm{H})$, dependendo se os valores estão acima ou abaixo dos limiares que definem os regimes, em conformidade com a equação $3 ; P_{t}$ e $A_{t}$ são, respectivamente, os preços recebidos pelo produtor e disponível no atacado da carne suína em $t, c$, $\alpha$ e $\beta_{i}$ são coeficientes da constante e dos preços estimados; $\varepsilon_{P, t}$ e $\varepsilon_{A, t}$ são os resíduos do modelo. Nesses resíduos, será feito o Teste Q de Ljung \& Box (1978), que irá identificar a presença de heteroscedasticidade, que determina a forte dispersão ao longo das séries de retorno.

Com base nos resíduos obtidos através do modelo TVEC, caso ocorra essa dispersão, analisa-se a matriz de covariância condicional com o modelo DCC-Garch para análise da razão dinâmica de hedge. A formulação matemática desse modelo é representada pela equação 4, sendo descrito como apresentada em Righi \& Ceretta (2014):

$$
\mathbf{H}_{t}=\boldsymbol{D}_{t}^{\prime} \boldsymbol{R}_{t} \boldsymbol{D}_{t} .
$$

Em que,

$\boldsymbol{D}_{t}=\operatorname{diag}\left(h_{1, t}^{1 / 2} \ldots h_{N, t}^{1 / 2}\right), h_{i, t}^{2}=c_{i}+b_{i} h_{i, t-1}^{2}+a_{i} \varepsilon_{i, t-1}^{2} ;$

$\boldsymbol{R}_{t}=\operatorname{diag}\left(q_{11, t}^{-1 / 2} \ldots q_{N N, t}^{-1 / 2}\right) \boldsymbol{Q}_{t} \operatorname{diag}\left(q_{11, t}^{-1 / 2} \ldots q_{N N, t}^{-1 / 2}\right) ;$

$\boldsymbol{Q}_{t}=(1-\alpha-\beta) \bar{Q}+\alpha u_{t-1} u_{t-1}^{\prime}+\beta \boldsymbol{Q}_{t-1}, u_{i, t}=\varepsilon_{i, t} / \sqrt{h_{i, t}} \sim$ skew $-t_{v} ;$

$\overline{\boldsymbol{Q}}$ é a matriz $N \times N$ composta pela variância incondicional de $u_{t} ; \alpha$ e $\beta$ são os coeficientes escalares não negativos satisfazendo $\alpha+\beta<1$; a definição de resíduos $u_{i, t}$ é estendida para o conceito multivariado através da distribuição multivariada $t$. Para obter o modelo que melhor se ajustasse foi empregue o Critério de Informação de Akaike (AIC).

Conforme Engle (2002), parâmetros do modelo Garch são estimados através de máxima verossimilhança em dois passos: i) no primeiro passo, a variância condicional é estimada por um modelo Garch univariado, e ii) no segundo passo, sendo dados os parâmetros do primeiro passo, são estimados os parâmetros para uma correlação 
condicional da volatilidade duas variáveis. Com base nas estimativas das matrizes de covariância condicional, é definida a seguinte razão de hedge ótima [5].

$\delta_{P A, t}=\frac{\operatorname{cov}\left(r_{P, t}, r_{A, t}\right)}{\operatorname{var}\left(\sigma_{A, t}^{2}\right)}$

Em [5], $\delta_{P A, t}$ é razão de hedge dinâmico entre o que é recebido pelo produtor $(\mathrm{P})$ e o que está disponível no atacado $(A)$ no instante $t ; \sigma_{A, t}^{2}$ é a variância dos valores contidos no atacado no instante $t, \operatorname{cov}\left(r_{P, t}, r_{A, t}\right)$ é a covariância entre os ativos no instante $t$.

Dentre alguns estudos na área, Müller (2007), por exemplo, buscou analisar a efetividade de estratégias de hedge, para o mercado de café arábica, em análise do mecanismo de mercados futuros da Bolsa de Mercadorias e Futuros (BM\&F). Os resultados indicaram que a estratégia mais eficiente foi a estratégia dinâmica para todos os contratos e que a cobertura completa não é considerada uma boa estratégia.

Ainda, a pesquisa de Bitencourt et al. (2006) buscou analisar dois modelos para cálculo das razões ótimas de hedge, nos preços do boi gordo, sendo o modelo convencional de regressão e o modelo Garch Bekk bivariado (BEKK é uma parametrização para o método Garch multivariado, desenvolvida por Baba, Engle, Kraft e Kroner), em que os resultados apontaram no sentido de que a razão ótima de hedge não é constante no tempo, sugerindo que a utilização de modelos que consideram a dependência temporal das séries seja mais realista.

Dentre esses estudos, com base no método anteriormente descrito e na literatura abordada, segue os resultados e discussões do presente estudo. Todos os testes e modelos apresentados nessa seção foram realizados no software $R$.

\section{Resultados e discussão}

Nos diversos elos da cadeia produtiva da carne suína (produção, comercialização, consumo, entre outros), o planejamento é fundamental para se obter ganhos ou se evitar perdas e, para tanto, conhecer as variações estacionais dos preços fornece importante subsídios de informações tanto aos produtores como aos atacadistas, visando a alocação mais eficiente dos recursos e dos produtos, assim como orienta os consumidores nas suas aquisições (Francisco, 1995).
Dessa forma, em estudos realizados pelos autores, percebe-se que, na evolução entre os preços recebidos pelo produtor e do atacado, conforme Figura 1, é possível denotar uma similaridade. Isso confirma a hipótese de Weydmann \& Seabra (2006), que verifica que o atacado é preço-líder do mercado da carne suína, e o produtor tende a seguir as oscilações de preços do atacado.

Conforme Thomé et al. (2013), eventos econômicos como o aumento substancial nos preços a partir de 2012, possivelmente ligados à redução de compras por parte do mercado russo e da abertura do mercado chinês para a carne suína nacional, bem como a fusão entre duas empresas líderes do mercado suíno, a regulamentação dos contratos de integração e o aumento do consumo de milho pelos EUA para alimentar seu programa de biocombustíveis, corroboram essa hipótese.

Dada a série de preços recebidos pelo produtor e disponíveis no atacado da cadeia produtiva estudada, verifica-se a presença de estacionaridade, a fim de determinar uma possível cointegração entre esses ativos. Para tanto, realiza-se o teste ADF na Tabela 1.

Conforme Tabela 1, o teste ADF identifica que os preços do atacado e do produtor não são estacionários, denotando a presença de raiz unitária, com valores não significativos ao nível de 5\% na estatística de p-valor de Hansen \& Seo (2002). Contudo, os retornos desses preços se tornaram estacionários e com p-valor significativo. Além disso, os resíduos na relação entre o preço recebido pelo produtor e o disponível no atacado também são estacionários, confirmando um relacionamento do

Figura 1. Preço recebido pelo produtor e disponível no atacado no período de $03 / 01 / 2011$ a 27/08/2015
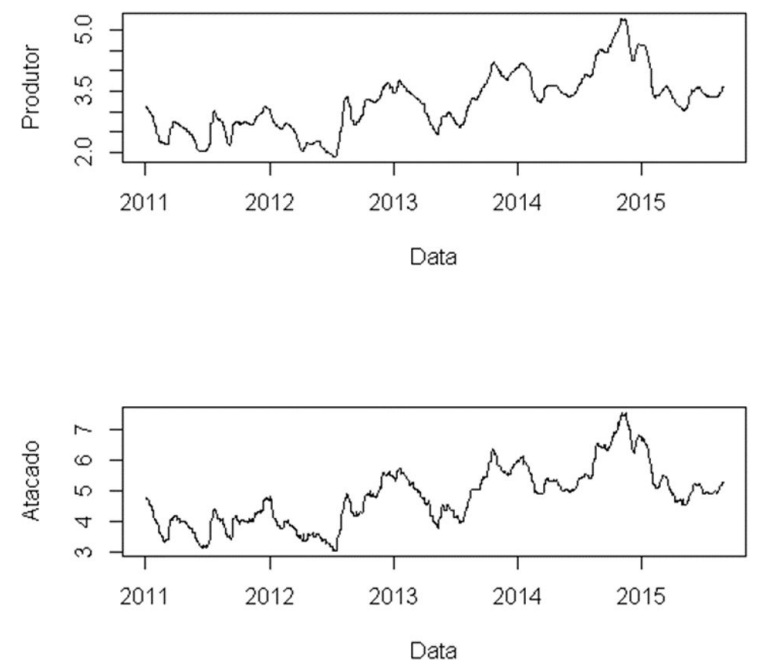

Fonte: Elaborado pelos autores. 
modelo de longo prazo, e possibilitando a execução de estratégias de hedge.

Ainda, as estimativas tradicionais de hedgeconsistem em definir uma alocação constante de um dado recurso no mercado futuro, a fim de minimizar os riscos inerentes às oscilações de preços do ativo. Desse modo, o agente financeiro aloca uma porcentagem de seus recursos em operações de hedge, mantendo-a constante durante toda a operação. $\mathrm{O}$ modelo de regressão linear, conforme Tabela 2, é o modelo exemplar que denota esse tipo de operação, em que considera apenas a proteção atrelada ao coeficiente linear do preço do atacado, que explica 49,94\% das informações contidas na relação com o preço recebido pelo produtor. As volatilidades, a correlação e a estratégia de hedge desse modelo são apresentadas nas linhas horizontais contínuas.

Tabela 1. Teste ADF para preço do produtor e atacado em nível e primeira diferença

\begin{tabular}{lcc}
\multicolumn{1}{c}{ Variável } & Estatística & p-valor \\
\hline Produtor(nível) & $-2,2548$ & 0,1870 \\
Atacado (nível) & $-2,5418$ & 0,1056 \\
Produtor(diferença) & $-8,8458$ & 0,0000 \\
Atacado (diferença) & $-8,7630$ & 0,0000 \\
Resíduos & $-6,7618$ & 0,0000 \\
\hline
\end{tabular}

Fonte: Elaborado pelos autores.

Tabela 2. Modelo Regressão Linear

\begin{tabular}{lcccc}
\hline \multirow{2}{*}{ Coeficiente } & \multicolumn{2}{c}{ Produtor } & \multirow{2}{*}{$\boldsymbol{R}^{2}$} & $\boldsymbol{R}^{2}$ ajustado \\
\cline { 2 - 3 } & Est. & p-valor & & \\
\hline Intercepto & 0,0000 & 0,8050 & $29,36 \%$ & $29,30 \%$ \\
Atacado & $\mathbf{0 , 4 9 9 4}$ & $\mathbf{0 , 0 0 0 0 *}$ & & \\
\hline
\end{tabular}

*Significante ao nível de $1 \%$

Fonte: Elaborado pelos autores.
Além disso, propõe-se na Tabela 3 uma estimação não linear de relacionamento entre os preços mediante o modelo TVEC, com regimes de alta e baixa volatilidade.

Na Tabela 3, seguindo a linha dos dados anteriormente apresentados, apresenta a predominância de dados pertencentes ao regime de alta $(84,1 \%)$, sendo o restante atrelado ao regime de baixa $(15,9 \%)$. Os regimes são representados por momentos de baixa e alta variação nos preços recebidos pelo produtor e nos praticados no atacado. No regime de baixa, ocorre transmissão entre os valores, em que observa-se o impacto de ambos na formação do preço, denotando cointegração entre os valores.

Por outro lado, o mesmo comportamento não se dá ao trabalhar com regimes de altas variações nos preços, tais como crises financeiras, em que a volatilidade do mercado é alta e a liquidez é baixa. Aqui, apenas ocorre a transmissão de valor estatisticamente significativa do atacado sobre a cotação ao produtor, o que implica que choques maiores que o threshold podem ser eliminados ao longo do tempo, aumentando a eficácia da estratégia de hedge. Isso corrobora com o trabalho de Weydmann \& Seabra (2006), que verifica que o preço praticado no atacado é considerado preço-líder dentro da cadeia produtiva da carne suína ao impactar no valor recebido pelo produtor e o que é praticado no varejo.

Para realizar uma estratégia dinâmica para o hedge dinâmico, identifica-se a presença de heteroscedasticidade dos resíduos do modelo TVEC, conforme Tabela 4.

Na Tabela 4, a maior parte das defasagens dos resíduos do preço recebido pelo produtor como no atacado mostrou-se estatisticamente significativa. Esse resultado confirma a presença do efeito heteroscedástico

Tabela 3. Modelo TVEC estimado para relações entre preços recebidos pelo produtor e atacado

\begin{tabular}{|c|c|c|c|c|}
\hline \multirow{2}{*}{ Coeficiente } & \multicolumn{2}{|c|}{ Regime de Baixa $(15,9 \%)$} & \multicolumn{2}{|c|}{ Regime de Alta $(84,1 \%)$} \\
\hline & Produtor & Atacado & Produtor & Atacado \\
\hline ECT & 0.0171 & 0.0263 & 0.0011 & 0.0066 \\
\hline Constante & 0.0060 & 0.0042 & -0.0004 & -0.0001 \\
\hline Produtor_1 & 0.2332 & 0.2641 & 0.2579 & 0.4292 \\
\hline Atacado_1 & 0.1120 & 0.1097 & 0.0560 & -0.0797 \\
\hline Produtor_2 & 0.4616 & 0.2198 & 0.0948 & 0.1684 \\
\hline Atacado_2 & 0.0913 & -0.0604 & 0.0529 & -0.0506 \\
\hline \multicolumn{5}{|c|}{$\begin{array}{l}\text { Hansen \& Seo (2002) Estatística p-valor } \\
67.6726<0,02\end{array}$} \\
\hline
\end{tabular}

Em negrito valores significativos ao nível de 5\%

Fonte: Elaborado pelos autores. 
Uma cadeia produtiva mais integrada? A utilização do hedge dinâmico na oscilação dos preços diários da cadeia produtiva da 526 carne suína

condicional, sugerindo que a volatilidade dos resíduos pode ser modelada. Desse modo, utilizando os resíduos obtidos através do modelo TVEC, foi estimada a matriz de covariância condicional com um modelo DCC-Garch. A formulação matemática do modelo foi representada pela Equação 4 e os resultados são apresentados na Tabela 5.

Conforme Tabela 5, as volatilidades dos preços recebidos nos produtos e os praticados pelo mercado foram afetadas por volatilidades passadas ao nível de $5 \%$ de significância. Esta característica é evidenciada pela significância estatística do parâmetro $\beta_{1}$. Adicionalmente, a volatilidade também demonstrou ser influenciada por choques quadráticos não antecipados em suas taxas de retorno, como pode ser notado pela significância estatística do parâmetro $\alpha_{1}$. Além disso, a filtragem dos

Tabela 4. Teste Q de Ljung \& Box (1978) nos resíduos do Modelo TVEC

\begin{tabular}{crr}
\hline \multirow{2}{*}{ Lag } & \multicolumn{2}{c}{ Estatística } \\
\cline { 2 - 3 } & Produtor & Atacado \\
\hline 1 & 1.753139 & 0.5942288 \\
2 & 8.836588 & 4.2117900 \\
3 & 9.799516 & 10.4267081 \\
4 & 15.045926 & 10.7112749 \\
5 & 98.894126 & 29.6890787 \\
6 & 101.350118 & 29.7829387 \\
7 & 101.406725 & 29.8019809 \\
8 & 102.623636 & 34.1901956 \\
9 & 102.629746 & 34.2658338 \\
10 & 103.561476 & 34.5232559 \\
\hline
\end{tabular}

Em negrito valores significativos ao nível de 5\% Fonte: Elaborado pelos autores. resíduos mediante distribuição t-student possibilitou bom ajustamento das variáveis ao modelo de volatilidade, conforme parâmetro Shape.

Dessa forma, a distribuição joint, que remete ao impacto conjunto da volatilidade dos preços foi estatisticamente significativa, indicando um bom ajustamento dos dados ao modelo DCC-Garch, e a qualidade deste quanto ao grau de explicação da volatilidade no mercado da carne suína.

Assim, na Figura 2 e 3 são apresentadas as séries das volatilidades dos preços, sua correlação dinâmica e razão de hedge ótima obtida através do modelo DCC-Garch. A linha que corta os valores representa a volatilidade, correlação e razão de hedge por meio da regressão linear.

Figura 2. Volatilidades dos preços (Produtor e Atacado) de 03/01/2011 a 27/08/2015.
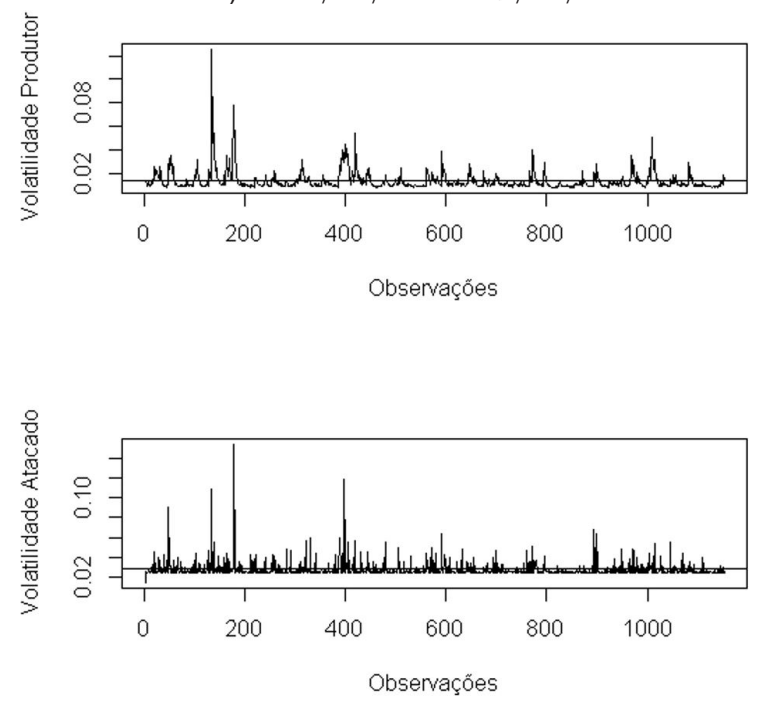

Fonte: Elaborado pelos autores.

Tabela 5. Modelo DCC-Garch estimado para os preços spot e futuro da soja

\begin{tabular}{|c|c|c|c|c|}
\hline \multirow{2}{*}{ Coeficiente } & \multicolumn{2}{|c|}{ Produtor } & \multicolumn{2}{|c|}{ Atacado } \\
\hline & Est. & p-valor & Est. & p-valor \\
\hline$\mu$ & -0.0005 & 0.0049 & -0.0002 & 0.1162 \\
\hline$\omega$ & 0,0000 & 0.0228 & 0.0006 & 0.0000 \\
\hline$\alpha_{1}$ & 0.3961 & 0,0000 & 0.9081 & 0.0040 \\
\hline $\boldsymbol{\beta}_{1}$ & 0.6029 & 0,0000 & 0.0919 & 0.0000 \\
\hline Shape & 2.3708 & 0,0000 & 2.1000 & 0.0000 \\
\hline Joint $_{\text {dcca } 1}$ & 0.0260 & 0.0157 & & \\
\hline Joint $_{d c c b 1}$ & 0.8245 & 0,0000 & & \\
\hline Joint $_{\text {mshape }}$ & 4.0000 & 0,0000 & & \\
\hline
\end{tabular}

Fonte: Elaborado pelos autores. 
De modo geral, os resultados da Figura 2 indicam que as volatilidades dos preços recebidos pelo produtor e utilizados no atacado apresentam diversos clusters de volatilidade ao longo do tempo, denotando momentos de forte instabilidade por longos períodos de tempo. Grandes oscilações nos preços praticados no atacado, decorrentes de fatores econômicos, como inflação e taxa de juros, bem como custos de transação, que afetam diretamente a cadeia produtiva da carne suína, provocam desequilíbrios que atingem quem oferta o produto bem como a demanda do mercado.

No que diz respeito à evolução da correlação dinâmica estimada entre os preços, a Figura 3 ilustra um comportamento estável ao longo do tempo, com maior correlação em períodos de maior volatilidade entre as variáveis. No entanto, há uma forte oscilação na correlação entre as observações 120 a 200, pertencentes ao período de julho a outubro de 2011, em que se constatou maior volatilidade desses preços no mercado, marcado principalmente pelos embargos dos governos russo e argentino, atingindo diretamente toda a conjuntura de

Figura 3. Correlação dinâmica e razão de hedge ótima, obtidos através do modelo DCC-Garch no período de 03/01/2011 a 27/08/2015.
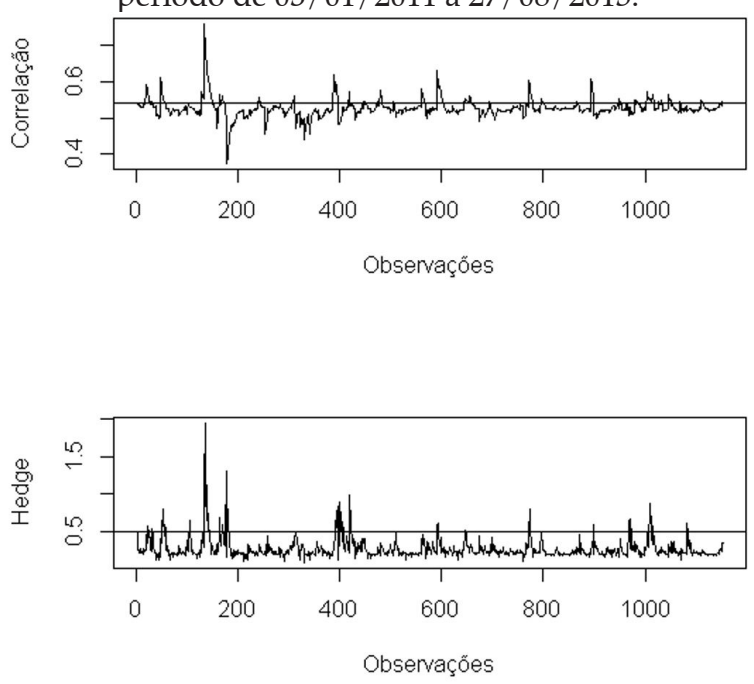

Fonte: Elaborado pelos autores.

Tabela 6. Efetividade do hedge no mercado de carne suína

\begin{tabular}{cc}
\hline Efetividade & Redução da Variância \\
\hline Regressão Linear & $29,36 \%$ \\
GARCH-DCC & $34,86 \%$ \\
\hline
\end{tabular}

Fonte: Elaborado pelos autores. um mercado estável. Além disso, na maior parte do período analisado as correlações ficam entre 0,37 e 0,78, indicando forte e relevante oscilação entre os mercados.

Ainda, como demonstrado pela Figura 3, essa alocação ótima de recursos não é constante ao longo do tempo, apresentando grande variabilidade. Dessa forma, a razão de hedge estática, oriunda do modelo linear não é satisfatória. Assim, calculou-se a razão ótima de hedge dinâmica, em que ajusta a proteção conforme o nível de oscilações nos preços do mercado.

Se comparado com as volatilidades dos preços, verifica-se que o maior percentual de proteção se dá justamente nas observações identificadas com clusters de volatilidade, e consequente instabilidade dos preços. Além disso, percebe-se que o hedge estático superestima a proteção nos períodos de menor turbulência no mercado, prejudicando uma maior lucratividade do produtor na venda para o atacado.

Assim, por meio do hedge dinâmico, possibilita-se uma nova forma de o produtor negociar seu produto considerando um impacto não constante do preço determinado no mercado atacadista, em que aumenta a efetividade de sua proteção contra as oscilações do mercado, conforme Tabela 6.

Conforme Tabela 6, as informações modeladas pela regressão linear conseguem reduzir as oscilações dos preços em $29,36 \%$. No entanto, com a utilização do modelo Garch-DCC, essa redução é de $34,86 \%$, significativamente maior comparado à proteção constante do modelo anterior.

\section{Conclusão}

Há disparidade notória na prática em dois dos principais elos da cadeia produtiva da carne suína, produtores e atacadistas, ocasionando barreiras e desequilíbrio dos preços a longo prazo. É nesse intento que o presente estudo centra esforços, de possibilitar aos suinocultores, por meio da realização de estratégia dinâmica de hedge, informações dos custos de produção e proteger-se das oscilações dos preços do atacado da carne suína e, com tais informações, obter maior lucratividade.

Ao analisar os preços, percebe-se que há grandes oscilações nos preços praticados no atacado, decorrente possivelmente de fatores econômicos, como inflação e taxa de juros, bem como de custos de transação que afetam diretamente a cadeia produtiva, provocando 
desequilíbrios que atingem quem oferta o produto e a demanda do mercado.

Nesse sentido, ao realizar a estimação TVEC para encontrar um equilíbrio de longo prazo em diferentes regimes de volatilidade, verifica-se cointegração em períodos de baixa volatilidade. Porém, quando há um aumento da volatilidade, apenas o valor disponível no atacado afeta o preço do produtor, corroborando com a ideia de preço-líder, e valoriza-se a realização de uma estratégia de hedge para proteção contra estas oscilações.

Na determinação da estratégia de hedge, informações modeladas pela regressão linear conseguiram reduzir as oscilações dos preços em $29,36 \%$ e, com a utilização do modelo Garch-DCC, essa redução foi de 34,86\%, otimizando as percepções e impulsionando a efetividade dos processos de planejamento, de ambos os elos estudados, em vista de as oscilações serem mais bem reduzidas e mensuradas. Tais dados trazem implicações econômicas importantes, em vista de fomentar uma perspectiva importante para a tomada de decisão.

Tais fatos levam a acreditar que o presente estudo tenha atingido sua contribuição ao apresentar dados relevantes quanto ao setor da suinocultura, possibilitando vantagens competitivas tanto aos produtores como aos atacadistas e, sobretudo, solucionando os entraves da cadeia. A utilização do hedge dinâmico poderia criar uma homogeneidade maior na capacidade de mensurar os preços, elevando o grau de competição do mercado e, principalmente, propiciar a formação de um mercado de futuros com ajustamentos diários de preços.

Para estudos futuros, possibilita a aplicabilidade deste paperjunto às outras regiões do País, para maior entendimento da cadeia produtiva ora apresentada, seu comportamento ante o mercado local e as competências organizacionais dos atores. Além disso, recomenda-se a realização da estratégia de hedge dinâmico em outras cadeias, ou mesmo na inter-relação entre diferentes cadeias produtivas, a fim de apresentar resultados relevantes ao mercado nacional e oferecer subsídios para a otimização dos processos e na tomada de decisões.

\section{Referências}

Arêdes, A. F., Santos, M. L., \& Gomes, M. F. M. (2012). Uma análise da transmissão de preços da carne suína em mercados selecionados no brasil no período de 2000 a 2009. Organizações Rurais \& Agroindustriais, 14(1), 142-154.

Balke, N. S., \& Fomby, T. B. (1997). Threshold Cointegration. International Economic Review, 38(3), 627-645.

Barcellos, M. D. (2012). Consumers' values and attitudes and their relation to the consumption of pork products: a study from A-Pork Chains in Brazil. Journal on Chain and Network Science, 12(1), 41-54.

Barcellos, M. D. (2011). Pork consumption in Brazil: challenges and opportunities for the Brazilian pork production chain. Journal on Chain and Network Science, 11(2), 99-113.

Bassi, N. S. (2008). O perfil dos suinocultores e avicultores de base familiar do sul do Brasil e seus principais canais de acesso a informações agropecuárias. Concórdia: Embrapa Suínos e Aves.

Bitencourt, W. A., Silva, W. S., \& Sáfadi, T. (2006). Hedge dinâmico: uma evidência para os contratos futuros brasileiros. Organizações Rurais e Agroindustriais, 8, 71-78.

Engle, R. F. (2002). Dynamic conditional correlation: A simple class of multivariate generalized autoregressive conditional heteroskedasticity models. Journal of Business \& Economic Statistics, 20(3), 339-350.

Engle, R. F., \& Granger, C. W. J. (1987). Co-integration and error correction: representation, estimation and testing. Chicago. Econometrica, 55(2), 251-276.

Francisco, V. L. F. (1995). Sazonalidade em séries temporais econômicas: aplicações. Agricultura em São Paulo, SP, 42(1), 57-71.

Gil, A. C. (2010). Como elaborar projetos de pesquisa (5. ed.). São Paulo: Atlas.

Guimarães, D., Amaral, G., Maia, G., Lemos, M., Ito, M., \& Custodio, S. (2017). Suinocultura: estrutura da cadeia produtiva, panorama do setor no Brasil e no mundo e o apoio do BNDES (n. 45). Rio de Janeiro: BNDES Setorial.

Griffith, G. R., \& Piggott, N. E. (1994). Asymmetry in beef, lamb and pork farm-retail price transmission in Australia. Agricultural Economics, 10(3), 307-316.

Hansen, B. E., \& Seo, B. (2002). Testing for two-regime threshold cointegration in vector error-correction models. Journal of Econometrics, 110(2), 293-318.

Hayenga, M. L., Jiang, B., \& Lence, S. H. (1996). Improving wholesale beef and pork product cross hedging. Agribusiness, 12(6), 541-559.

Ljung, G. M., \& Box, G. E. P. (1978). On a measure of lack of fit in time series models. Biometrika, 65(2), 297-303.

Martins, A. P. (2010). Transmissão de preços na cadeia produtiva de suínos (Tese de doutorado). Universidade Federal de Viçosa, Viçosa, MG. 
Miele, M. (2006). Contratos, especialização, escala de produção e potencial poluidor na suinocultura de Santa Catarina. (Tese de doutorado) Universidade Federal do Rio Grande do Sul, Porto Alegre.

Müller, C. A. S. (2007). Effectiveness analysis of statics and dynamics hedge strategies for Brazilian arabian coffee market 5 p. (Tese de doutorado). Universidade Federal de Viçosa, Viçosa.

Raimundo, L. M. B., \& Batalha, M. O. (2015). Swine meat market in Sao Paulo: segments and strategies. Gestão \& Produção, 22(2), 391-403.

Righi, M. B., \& Ceretta, P. S. (2014). Transmissão da volatilidade entre ações de grandes e pequenas empresas no mercado brasileiro de capitais. Revista de Administração da UFSM, 7(2), 249-264.

Rocha, D. T. (2006). Competitividade entre os sistemas integrado e independente de produção de suínos (Dissertação de mestrado). Universidade Federal de Viçosa, Viçosa.

Rodrigues, G. Z., Gomes, M. M., Cunha, D. A., \& Santos, V. F. (2015). Evolução da produção de carne suína no brasil: uma análise estrutural-diferencial. Revista de Economia e Agronegócio - REA, 6(3), 343-366.

Rodríguez, S. V., Plà, L. M., \& Faulin, J. (2014). New opportunities in operations research to improve pork supply chain efficiency. Annals of Operations Research, 219(1), 5-23.

Santini, G. A., \& Souza Filho, H. M. (2004, julho 25-28). Mudanças tecnológicas em cadeias agroindustriais: uma análise dos elos de processamento da pecuária de corte, avicultura de corte e suinocultura. In Anais do $42^{\circ}$ Congresso Brasileiro de Economia e Sociologia Rural. Cuiabá: Congresso Brasileiro de Economia e Sociologia Rural.

Santos, A. H. G. (2001). Potencialidade de implantação do contrato futuro de suínos no Brasil (Tese de doutorado). Universidade Federal de Viçosa, Viçosa.

Santos Filho, J. I. (2008, julho 20-23). Formação de preços, rentabilidade e concentração no mercado de suinícola brasileiro. In 46th Congress. Rio Branco, Acre: Sociedade Brasileira de Economia, Administração e Sociologia Rural (SOBER).

Stigler, M. (2010). Threshold cointegration: overview and implementation in $R$ (Working Paper). Revision 5.

Thomé, K. M., Reis, R. P., \& Paiva, F. D. (2013). BrazilRussia meat market: an analysis from the perspective of the new instituional economics. Organizações Rurais \& Agroindustriais, 15(1), 75-86.

Tong, H. (1990). Non-Linear Time Series: a dynamical system approach. Oxford: Oxford Science Publications.

Weydmann, C. L., \& Seabra, F. (2006). Transmissão de preços na cadeia de carne suína: uma aplicação para os preços de São Paulo. Revista de Economia e Agronegócio - REA, 4(3), 269-288.

Villwock, L. H. M. (2001). Consórcios Agroexportadores: Estratégia para o desenvolvimento competitivo da cadeia de produção de suínos no RS. (Tese de doutorado). Universidade Federal do Rio Grande do Sul, Porto Alegre, RS. 